\title{
OMENTOBURSECTOMÍA EN CÁNCER GÁSTRICO: CONTROVERSIAS ACTUALES*
}

\author{
Drs. Attila Csendes J. ${ }^{1}$, Jaime Matus R. ${ }^{1}$, Marcelo Zamorano D. ${ }^{1}$, Galo Cárdenas S. ${ }^{1}$ \\ 1 Departamento de Cirugía, Hospital Clínico Universidad de Chile. \\ Santiago, Chile.
}

\section{Abstract}

\section{Bursectomy and omentectomy in the surgical treatment of gastric cancer}

There is controversy about the usefulness of bursectomy and omentectomy during the surgical treatment of gastric cancer. Bursectomy, a procedure dissecting the peritoneal lining covering the pancreas and the anterior plane of the transverse mesocolon, is a major surgical aggression. There is no evidence that the procedure improves five years survival. It may be useful in serosal gastric cancer of the posterior wall. Excision or resection of all or part of an omentum does not have either an effect on survival. However, we recommend its use since its lack of benefit has not been completely ascertained.

Key words: Bursectomy, omentectomy, gastric cancer.

\section{Resumen}

Dentro de las etapas quirúrgicas de la gastrectomía por cáncer gástrico, existen controversias respecto a la bursectomía y la omentectomía. La bursectomía (resección de peritoneo que cubre la porción anterior del mesocolon transverso y la cápsula pancreática) representa una mayor agresión quirúrgica, sin evidencia actual de que aumenta la sobrevida a 5 años, ello evaluado en 3 estudios clínicos. Su utilidad parece estar en pacientes con cáncer gástrico seroso de la cara posterior. La omentectomía total, comparada con la omentectomía parcial, ha sido evaluada más extensamente, sin poder demostrar hasta la actualidad la superioridad de uno u otro procedimiento en cuanto a sobrevida a 5 años. Sin embargo, mientras no se demuestre claramente lo opuesto en estudios científicos, recomendamos realizarla rutinariamente.

Palabras clave: Cáncer gástrico, omentectomía, bursectomía.

En la década de 1960, gracias a los trabajos del Dr. Jinnai D. et al. La bursectomía se constituyó como un elemento integral del manejo quirúrgico radical del cáncer gástrico, con la finalidad de eliminar completamente micro metástasis presentes en la trascavidad de los epiplones y la enfermedad linfonodal peri pancreática, al lograr la remoción en bloque de dicha cavidad, disminuyéndose así la recurrencia local de la enfermedad ${ }^{1}$. La bursectomía consiste en la omentectomía más resección del peritoneo que recubre la porción anterior del mesocolon transverso y la cápsula pancreática.

\footnotetext{
*Recibido el 18 de agosto de 2014 y aceptado para publicación el 12 de mayo de 2015.
}

Los autores no refieren conflictos de interés.

Correspondencia: Dr. Attila Csendes J.

acsendes@hcuch.cl 
En la actualidad las guías clínicas de la Japanese Gastric Cancer Association recomiendan la bursectomía como un procedimiento mandatorio en el tratamiento quirúrgico del cáncer gástrico con invasión a la serosa ${ }^{2}$. Sin embargo, recientes estudios han cuestionado el beneficio real de este procedimiento quirúrgico al tratarse la bolsa omental de un espacio abierto con comunicación libre al resto de la cavidad abdominal por medio del hiato de Winslow.

El omento o epiplón mayor es una estructura anatómica conocida desde la antigüedad y a la que se le han atribuido diversas funciones, tanto místicas como fisiológicas. Los antiguos embalsamadores egipcios por ejemplo lo usaban para leer presagios al observar sus variaciones anatómicas ${ }^{3}$, para Galeno tenía una función reguladora de la temperatura corporal $^{4}$, en la actualidad se considera que juega un rol preponderante en los mecanismos de defensa peritoneal lo que le otorga el papel del policía de la cavidad peritoneal ${ }^{5,6}$.

\section{Reseña anatomofisiológica}

Las cuatro funciones principales del omento como mecanismo de defensa se centran en: a) su capacidad de adherirse a los sitios de inflamación (movimiento facilitado por la peristasis intestinal); b) absorción de bacterias y cuerpos extraños; c) a su capacidad para proveer leucocitos con lo que se logra una respuesta inmune $\operatorname{local}^{6} \mathrm{y}$ d) su capacidad para promover la angiogénesis en las estructuras contiguas?

En 1874, Ranviere describe la presencia de conglomerados leucocitarios en espacios perivasculares del omento, a los que actualmente denominamos milky spots ${ }^{8}$. Estos están constituidos por una fina red de fibras reticulares, leucocitos y capilares fenestrados conformando glomérulos omentales los cuales están especialmente adaptados para facilitar la migración leucocitaria9.

Desde los trabajos realizados por Dux en 1969 y Green y Williams en 1978, se sabe que los milky spots pueden ser infiltrados por células neoplásicas en etapas tempranas de la carcinomatosis peritoneal en modelos animales. Posteriormente, en 1995 Tsujimoto demuestra en modelo animal los mismos resultados ${ }^{10}$. Aunado a lo anterior Yamagata et al, en 1998, publican un trabajo en el cual demuestran que la obstrucción de los conductos linfáticos por infiltración neoplásica juega un rol importante en la diseminación neoplásica intraperitoneal y en las metástasis hematógenas por la presencia de reflujo linfático y cambios en el flujo linfovenoso ${ }^{11}$.

En diversos modelos animales se ha demostrado que la omentectomía tiene un impacto en la supervi- vencia de células neoplásicas libres intraperitoneales y disminuye la incidencia de recurrencia local. Esto y el hecho de que el omento se encuentra íntimamente relacionado con el estómago y el drenaje linfático gástrico, hacen que la omentectomía sea parte integral de la gastrectomía radical en cáncer gástri$\mathrm{co}^{12-14}$. Sin embargo, no existe evidencia científica clara sobre la extensión de la resección omental ${ }^{15,16}$.

La trascavidad de los epiplones, también llamada bolsa omental o bursa omentalis, corresponde a un espacio virtual de forma irregular comprendido entre el estómago, el páncreas y ambos omentos. Se considera como un gran divertículo de la cavidad peritoneal con la cual se encuentra comunicada a través del Hiato de Winslow. Se distinguen dos porciones separadas entre sí por el foramen de la bursa omentalis, cuyos límites anatómicos son los repliegues peritoneales de la arteria hepática y la arteria gástrica izquierda. La trascavidad propiamente dicha se extiende desde el foramen de la bursa omentalis hasta el hilio esplénico, el epiplón gastroesplénico y pancreatoesplénico, con prolongaciones a los espacios subhepático, esplénico y colónico (Figuras 1 y 2), cuyos límites anatómicos podemos apreciar en la Tabla 1.

\section{Controversias actuales en bursectomía}

Existen dos factores indispensables para que la bursectomía sea aceptada como tratamiento es-

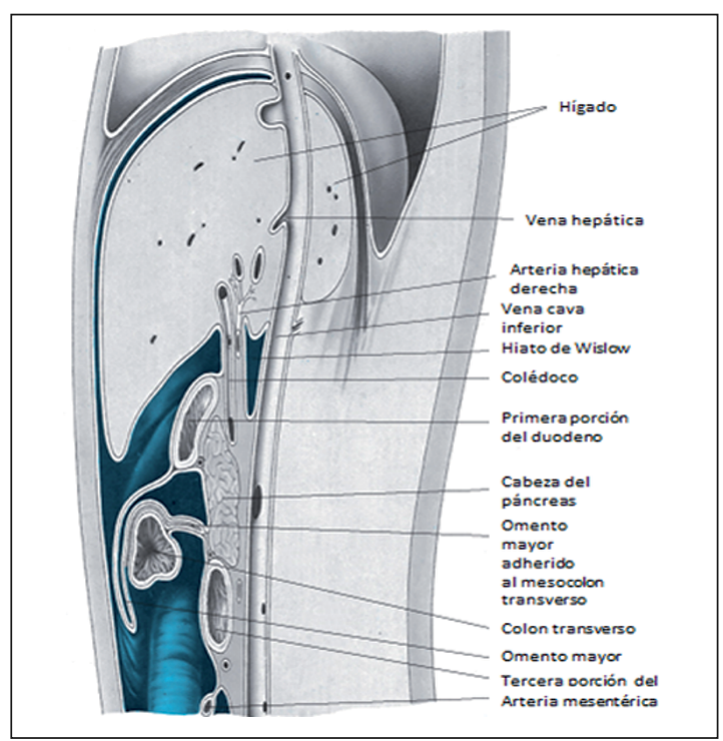

Figura 1. Corte sagital del abdomen que muestra el hiato de Wislow y la trascavidad de los epiplones. Tomado y adaptado de ElsevierLtd 2005. Standring: Gray'sAnatomy $39 \mathrm{e}-$ www.graysanatomyonline.com 


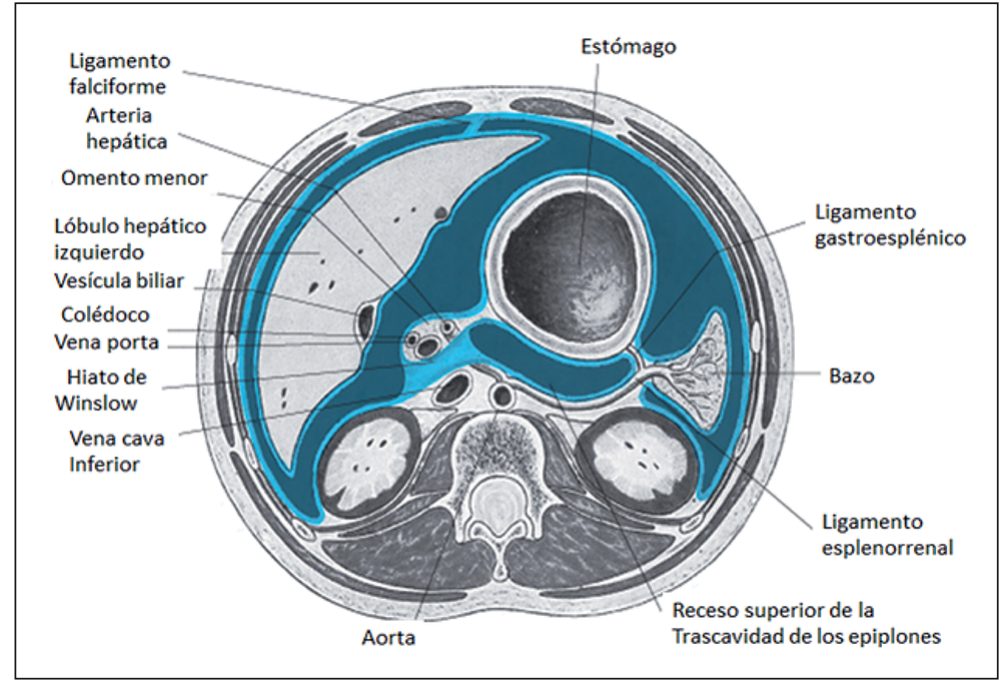

Figura 2. Corte axial del abdomen que muestra el hiato de Winslow y la trascavidad de los epiplones. Tomado y adaptado de ElsevierLtd 2005. Standring: Gray'sAnatomy 39e-www. graysanatomyonline.com

Tabla 1. Porciones y límites anatómicos de la Trascavidad de los epiplones

\begin{tabular}{|llll|}
\hline Límite & Hiato de Wislow & Vestíbulo & Trascavidad propiamente dicha \\
\hline Anterior & Pedículo hepático & Omento menor & $\begin{array}{l}\text { Cara gástrica posterior y porción gas- } \\
\text { trocólica del omento mayor }\end{array}$ \\
Posterior & Vena cava inferior & $\begin{array}{l}\text { Peritoneo parietal que cubre } \\
\text { la porción celíaca }\end{array}$ & $\begin{array}{l}\text { Peritoneo que cubre el cuerpo y cola } \\
\text { del páncreas, suprarrenal y riñón } \\
\text { izquierdo }\end{array}$ \\
Superior & Segmento hepático I & $\begin{array}{l}\text { Ligamento coronario del } \\
\text { hígado }\end{array}$ & $\begin{array}{l}\text { Reflexión peritoneal desde el estóma- } \\
\text { go al diafragma }\end{array}$ \\
${ } }$ & $\begin{array}{l}\text { Reflexión peritoneal desde la vena } \\
\text { cava inferior al borde superior de la } \\
\text { primera porción del duodeno }\end{array}$ & Cabeza del páncreas & $\begin{array}{l}\text { Hoja posterior de la porción gastro- } \\
\text { cólica del omento mayor adosada al } \\
\text { mescolon transverso }\end{array}$ \\
\hline
\end{tabular}

tándar del cáncer gástrico: seguridad y beneficio oncológico.

Yoshitaka et $\mathrm{al}^{2}$, en 2007, señalan que las células neoplásicas raramente están confinadas exclusivamente a la bursa omentalis, encontrándose en esta localización exclusiva y únicamente en el 32,6\% de los casos con lavado peritoneal positivo para células neoplásicas, correspondiendo al 10,3\% del total de casos de su serie, lo que contrasta con el 79,1\% y el $39,5 \%$ de casos con células neoplásicas detectadas en el saco de Douglas y el espacio subfrénico izquierdo respectivamente, en pacientes con lavado peritoneal positivo. Este hecho nos orienta a pensar que la bursectomía probablemente tenga un rol limitado en el tratamiento radical del cáncer gástrico, siendo los pacientes con afección tumoral serosa de la pared gástrica posterior, el grupo de pacientes más beneficiados con este procedimiento.
Desde que se propuso la implementación de la bursectomía como elemento integrante del tratamiento quirúrgico radical del cáncer gástrico, se ha suscitado polémica debido a que es un procedimiento que demanda mayor tiempo quirúrgico y conlleva complicaciones, tales como: fistula pancreática, lesión vascular y/u obstrucción intestinal como lo señalan Boulas et $\mathrm{al}^{17}$, en un trabajo publicado en 2013, donde reporta que la bursectomía se asoció a un tiempo quirúrgico adicional de $41 \mathrm{~min}$, sangrado adicional de $65 \mathrm{ml}$, morbilidad postoperatoria de $19,4 \%$, fístula pancreática en $4,2 \%$, obstrucción intestinal en $11,1 \%$, íleo prolongado en 4 casos y síndrome de asa aferente en un caso, con una mortalidad hospitalaria de $1,4 \%$, sin que existiese diferencia estadísticamente significativa en este parámetro al comprarse con gastrectomía D2 sin bursectomía.

Imamura et $\mathrm{al}^{18}$, en el año 2011, publican los 
Tabla 2. Morbimortalidad en bursectomía

\begin{tabular}{|lccccc|}
\hline & \multicolumn{2}{c}{ Morbilidad \% } & \multicolumn{2}{c|}{ Mortalidad \% } \\
Autor & Bursectomía (+) & Bursectomía (-) & Bursectomía (+) & Bursectomía (-) \\
\hline Eom BW et al & 24,3 & 26,5 & 0 & 0,3 \\
Kochi M et al & 24 & 25,6 & 0 & 0,8 \\
\hline Fujita J et al* & 14,3 & 14,3 & 0,95 & 0,95 \\
\hline Imamura H et al* & 14,3 & 14,3 & 0,95 & 0,95 \\
\hline
\end{tabular}

*Estudios realizados por el mismo grupo de autores.

resultados de un ensayo clínico controlado aleatorizado en el que se incluyeron 210 pacientes con cáncer gástrico T2-T3, provenientes de 11 hospitales japoneses, encontrando que la bursectomía se asocia a un tiempo operatorio promedio 26 min mayor que en el grupo en el que no se realizó bursectomía, una morbilidad operatoria de $14,3 \%$ en ambos grupos, no apreciándose diferencias significativas en cuanto a fistula pancreática, hemorragia postoperatoria, obstrucción intestinal, abscesos intraabdominales, ni días de estancia hospitalaria (Tabla 2).

\section{Sobrevida en bursectomía}

En 2004, Yoshikawa et al ${ }^{19}$, publican un estudio retrospectivo cuyo objetivo primario fue evaluar el impacto de la bursectomía en la sobrevida a 3 y 5 años, para lo que incluyeron 134 pacientes, en el que encuentran sobrevida de $67,3 \%$ y $53 \%$ a 3 y 5 años respectivamente en el grupo de pacientes con tumores con afección de la pared gástrica posterior y de $68,8 \%$ y 53,8 a los 3 y 5 años para el grupo de pacientes con tumores gástricos de localización indistinta, sin encontrar diferencia estadísticamente significativa entre ambos grupos $(p=0,969)$, señalando al tipo macroscópico del tumor y la invasión linfonodal como factores independientes de riesgo para sobrevida.

\section{Omentectomía y cáncer gástrico}

El tratamiento quirúrgico estándar del cáncer gástrico consiste en la gastrectomía, linfadenectomía de la primera y segunda barrera ganglionar más omentectomía, con la finalidad de resecar la enfermedad macro y microscópica con márgenes quirúrgicos libres $^{20,21}$. Dicho procedimiento constituye la única opción de curación en estos pacientes.

Contrario a lo sucedido con la linfadenectomía D2 en cáncer gástrico, donde se demostró beneficio oncológico, el papel de la omentectomía total en este contexto últimamente ha sido cuestionado en múltiples estudios ${ }^{22}$ y actualmente la Japanese Gastric Cancer Association recomienda la omentectomía parcial para tumores $\mathrm{T} 1$ y $\mathrm{T} 2$ y la omentectomía total únicamente para tumores $\mathrm{T} 3$ y $\mathrm{T} 4$ como procedimiento estándar ${ }^{21}$.

En 2003, Fujita et al, publican un estudio en el que comparan la gastrectomía con preservación omental con la gastrectomía convencional en cáncer gástrico avanzado sin encontrar diferencia significativa entre ambos grupos en los resultados a largo plazo y encuentran una mayor tasa de complicaciones en el grupo sometido a omentectomía. Un año después, Watanabe et al, publican un estudio con similares resultados ${ }^{23}$.

En 2013, Hasegawa et al, publican los resultados de una cohorte retrospectiva de 330 pacientes con adenocarcinoma gástrico T2-4, N0-3, M0, excluyéndose los pacientes con lavado peritoneal positivo para células neoplásicas, en que comparan la gastrectomía más omentectomía con la gastrectomía con preservación omental, encontrando sobrevida libre de enfermedad a 3 y 5 años de 72,9\% (IC 95\% rango de $64,1-81,7 \%$ ) y $66,2 \%$ (IC 95 rango del $56,6-75,8 \%$ ) respectivamente en el grupo con omentectomía y 76,7\% (IC 95\% rango 67,9-81,2\%) y $67,3 \%$ (IC $95 \%$ rango (55,1-79,5\%) en el grupo con preservación omental, sin evidenciar diferencia estadísticamente significativa entre ambos $(\mathrm{p}=0,750)$. Los principales sitios de recidiva tumoral fueron el peritoneo, con 7 casos en ambos grupos, el hígado con 7 casos en el grupo de gastrectomía más omentectomía y 8 casos en el grupo de gastrectomía más preservación omental, sin encontrarse diferencias estadísticamente significativas $(\mathrm{p}=0,863)^{23}$.

Kim et al, en 2014, reportan los resultados de una cohorte retrospectiva en la que incluyen 146 pacientes con cáncer gástrico avanzado (T2 y T3) (se excluyeron todos los casos con invasión serosa), sometidos a gastrectomía laparoscópica más omentectomía total (80 casos) y pacientes con gastrectomía laparoscópica más omentectomía parcial (66 casos), encontrando 65\% de tumores T3 en el 
Tabla 3. Patrón de recurrencia en omentectomía

\begin{tabular}{|llccccccc|}
\hline Autor & Grupo & $\begin{array}{c}\text { Recurrencia } \\
\text { \% }\end{array}$ & \multicolumn{5}{c|}{ Patrón de recurrencia } \\
& & & Hígado & Peritoneal & Linfonodal & Órganos & Remanente \\
distantes\% & gástrico \% \\
Hasegawa et al & Omentectomía total & 18,3 & 7,4 & 7,4 & 3,06 & 1,02 & - \\
& Omentectomía parcial & 20,4 & 8,16 & 7,4 & 2,04 & 3,06 & - \\
\multirow{2}{*}{ Kim et al } & Omentectomía total & 17,5 & 3,75 & 3,75 & 3,75 & 2,5 & 3,75 \\
& Omentectomía parcial & 7,5 & 1,51 & 3,02 & 1,51 & 1,51 & 1,51 \\
\hline
\end{tabular}

grupo sometido a omentectomía total y un 56,1\% de tumores T2 en el grupo sometido a omentectomía parcial. Con respecto a la recidiva tumoral, ésta se presentó en $17,3 \%$ y 7,6\% en el grupo sometido a omentectomía total y en el grupo de omentectomía parcial respectivamente. No se encontró diferencia entre ambos grupos en lo referente a sobrevida libre de enfermedad ${ }^{22}$.

En la Tabla 3 se aprecia el patrón de recurrencia presentado en pacientes sometidos a omentectomía parcial vs omentectomía total.

\section{Conclusiones}

Pese al desarrollo tecnológico suscitado en las últimas décadas así como al mayor conocimiento de la biología tumoral, el cáncer gástrico persiste dentro de las primeras causas de muerte a nivel mundial.

La cirugía del cáncer gástrico ha sufrido grandes cambios con el transcurso del tiempo pasando de las grandes resecciones radicales con extensas linfadenectomías a resecciones cada vez más limitadas llegando incluso a las resecciones endoscópicas en el caso del cáncer gástrico temprano. Durante este proceso evolutivo de la cirugía han surgido diversas interrogantes, como ser la necesidad quimioterapia neo adyuvante y/o adyuvante, o la necesidad de extensas resecciones llegándose a plantear en casos seleccionados la peritonectomía junto a la resección en bloque de los órganos involucrados, la extensión de la linfadenectomía y en la necesidad de la omentectomía total más bursectomía.

Diversos estudios han mostrado resultados contradictorios en lo referente a la utilidad de la bursectomía en el contexto del cáncer gástrico, al tratarse esta de una cavidad comunicada por el foramen de Wislow con la consecuente eventual diseminación de células neoplásicas al resto de la cavidad abdominal. Sin embargo, la evidencia científica actual no ha revelado resultados concluyentes que apoyen o descarten la utilidad de la bursectomía, razón por la que este procedimiento no debe ser abandonado y debiera ser evaluado con más estudios prospectivos.

Por otra parte, la omentectomía parcial parece presentar resultados oncológicos similares a la omentectomía total en cáncer gástrico, con una menor morbilidad y menor tiempo operatorio, principalmente en el contexto de la cirugía laparoscópica. Sin embargo, la evidencia científica no es concluyente en lo referente a resultados oncológicos por lo que parece prudente esperar resultados de estudios con mejor diseño metodológico antes de abandonar la práctica de la omentectomía total sobre todo en cáncer gástrico con afección de la serosa.

\section{Referencias}

1. Eom BW, Joo J, Kim YW, Bae JM, Park KB, Lee JH, et al. Role of bursectomy for advanced gastric cancer: Result of a case control study from a large volume hospital. EJSO 2013;39:1407-14.

2. Yamamura Y, Ito S, Mochizuki Y, Nakanishi H, Tatematsu M, Kodera Y. Distribution of free cancer cells in the abdominal cavity suggests limitations of bursectomy as an essential component of radical surgery for gastric carcinoma, Gastric Cancer 2007;10:24-8.

3. McLachlin A, Denton D. Omental protection of intestinal anastomoses. Am J Sung.1973;125:134-40.

4. Goldsmith HS. The omentum: Research and clinical applications. Springer-Verlag, New York, 1990.

5. Hall J, Heel K, Papadimitriou J, Platell C. The pathobiology of peritonitis. Gastroenterology1998;114:185-96.

6. Platell C, Cooper D, Papadimitriou JM, Hall JC. The omentum. World J Gastroenterol. 2000;6:169-76.

7. Cartier R, Brunette I, Hashimoto K, Bourne WM, Schaff HV. Angiogenic factor: a possible mechanism for neovascularization produced by omental pedicles. J Thoracic Cardiovascular Surg. 1990;99:264-8.

8. Vanvugt E, Van Rijthoven EAM, Kamperdijk EWA, Beelen RHJ. Omental milky spots in the local immune response in the peritoneal cavity of rats. Anatomical Record 1996;244:235-45. 
9. Mironov VA, Gusev SA, Baradi AF. Mesothelial stomata overlying omental milky spots: scanning electron microscopic study. Cell Tissue Research 1979;201:32730.

10. Tsujimoto H, Takhashi T, Hagiwara A, Shimotsuma M, Sakakura C, Osaki K, et al. Site-specific implantation in the milky spots of malignant cells in peritoneal dissemination: immunohistochemical observation in mice inoculated intraperitoneally with bromodeoxyuridinelabelled cells. Br J Cancer 1995;71:468-72.

11. Yamagata K, Kumagai K, Shimizu K, Masuo K, Nishida Y, Yasui A. Gastrointestinal Cancer Metastasis and Lymphogenous Spread: Viewpoint of Animal Models of Lymphatic Obstruction. Jpn J Clin OncoI. 1998;28:1046.

12. Lawrance RJ, Loizidou M, Cooper AJ, Alexander P, Taylor I. Importance of the omentum in the development of intra-abdominal metastases. Br J Surg. 1991;78:1179.

13. Weese JL, Ottery FD, Emoto SE. Does omentectomy prevent malignant small bowel obstruction. Clin Exp Metastasis 1988;6:319-24.

14. Kodera Y, Nakanishi H, Ito S, Yamamura Y, Kanemitsu Y, Shimizu Y, et al. Quantitative detection of disseminated cancer cells in the greater omentum of gastric carcinoma patients with real-time RT-PCR: a comparison with peritoneal lavage cytology. Gastric Cancer 2002,5:69-76.

15. Robertson CS, Chung SC, Woods SD, Griffin SM, Raimes SA, Lau JT, et al. A prospective randomized trial comparing R1 subtotal gastrectomy with R3 total gastrectomy for antral cancer. Ann Surg. 1994;220:176-
82.

16. Hagiwara A, Sawai K, Sakakura C, Shirasu M, Ohgaki $\mathrm{M}$, Yamasaki J, et al. Complete omentectomy and extensive lymphadenectomy with gastrectomy improves the survival of gastric cancer patients with metastases in the adjacent peritoneum. Hepato-Gastroenterology. 1998;45:1922-9.

17. Blouhos K, Boulas KA, Hatzigeorgiadis A. Bursectomy in gastric cancer surgery: surgical technique and operative safety. Updates in Surgery 2013; 65 :95-101.

18. Imamura H, Kurokawa Y, Kawada J, Tsujinaka T, Takiguchi S, Fujiwara Y, et al. Influence of Bursectomy on Operative Morbidity and Mortality After Radical Gastrectomy for Gastric Cancer: Results of a Randomized Controlled Trial. World J Surg. 2011;35:625-30.

19. Yoshikawa T, Tsuburaya A, Kobayashi O, Sairenji M, Motohashi H, Hasegawa S, et al. Is bursectomy necessary for patients with gastric cancer invading the serosa? Hepatogastroenterology 2004;51:1524-6.

20. Nakajima T. Gastric cancer treatment guidelines in Japan. Gastric Cancer 2002;5:1-5.

21. Japanese Gastric Cancer A: Japanese gastric cancer treatment guidelines 2010. Gastric Cancer 2011;14:11323.

22. Kim DJ, Lee JH, Kim W. A comparison of total versus partial omentectomy for advanced gastric cancer in laparoscopic gastrectomy World J of Surg Oncol. 2014;26:12-64.

23. Hasegawa S, Kunisaki C, Ono H, Oshima T, Fujii S, Taguri M, et al. Omentum-preserving gastrectomy for advanced gastric cancer: a propensity-matched retrospective cohort study. Gastric Cancer 2013;16:383-8. 